\title{
Soziale Netzwerke und europäischer Datenschutz
}

In den global im Internet nutzbaren Sozialen Netzwerken ${ }^{1}$ spiegelt sich das Dilemma des Datenschutzes deutlich wider: Auf Grundlage nationaler Regeln oder Gesetze versuchen einzelne Staaten auf den Umgang der Anbieter von Sozialen Netzwerken mit den persönlichen Daten ihrer Nutzer Einfluss zu nehmen. Das gilt auch für Europa, denn die inzwischen fast 15 Jahre alte Datenschutzrichtlinie 95/46/EC ist ja differenziert in den EUMitgliedsstaaten in geltendes Recht umgesetzt worden. Da die Prinzipien der Europäischen Richtlinie inzwischen auch in vielen anderen Regionen der Welt de facto anerkannt sind, kann es die Mühe lohnen, auf ihrer Basis Rahmenempfehlungen für den Datenschutz in Sozialen Netzwerken zu entwickeln.

Die europäische ,Artikel-29-Datenschutzgruppe' hat sich dieser Herausforderung gestellt und jüngst als WP $163 \mathrm{Opi-}$ nions 5/2009 on online social networking ${ }^{2}$ verabschiedet. Berücksichtigt wurden dabei insbesondere das Rome Memorandum $^{3}$ der Internationalen Arbeitsgruppe zu Datenschutz in der Telekommunikation und die Position der European Network and Information Security Agency (ENISA) Security Issues and Recommendations for Online Security Networks 4 .

Die Dienste von Anbietern Sozialer Netzwerke werden rechtlich als IT-Dienstleistungen behandelt. Sie zielen insbesondere darauf ab,

- die Nutzer zu motivieren persönliche

Daten für die Selbstdarstellung oder

1 Zu "Social Networks" siehe auch Gateway, DuD 1/2009, S. 53.

2 Stellungnahme vom 12.06.2009, siehe http:// ec.europa.eu/justice_home/fsj/privacy/docs/wpdocs /2009/wp163_de.pdf

3 Report and Guidance on Privacy in Social Network Services vom 04.03.2008, siehe http://www. datenschutz-berlin.de/attachments/461/WP_social_network_services.pdf

4 Recommendations for Online Social Networks vom 14.11.2007, siehe http://www.enisa.europa.eu/ doc/pdf/deliverables/enisa_pp_social_networks.pdf
Profilbildungen zur Verfügung zu stellen,

- Werkzeuge bereit zu stellen, mit denen die Nutzer ihre Daten (eigene Bilder, Tagebucheinträge usw.) an andere Webseiten posten und

- das Soziale Networking zu ermöglichen und durch Kontaktlisten für jeden Nutzer zu unterstützen.

Das Geschäftsmodell der Anbieter besteht u. a. darin, dass auf den vom Nutzer aufgerufenen Webseiten - auch abhängig vom Inhalt der vom Nutzer gesendeten Daten - Werbung für weitere Dienste oder Produkte angeboten wird. Je umfassender die Informationen über den Nutzer und sein Profil sind, desto attraktiver ist dieses Geschäftsmodell für den werbenden Geschäftspartner des Anbieters. Sowohl die Art der Dienstleistungen als auch das Geschäftsmodell signalisieren, dass sich der Nutzer einer Internet-Umgebung anvertraut, in der die kulturellen Qualitäten des Datenschutzes - Informationelle Selbstbestimmung und Bewahrung der Privatspäre - erkennbar Gefahren ausgesetzt sind.

Der Wert der von der Artikel-29-Gruppe erarbeiteten Stellungnahme besteht darin, dass die Anwendbarkeit der Definitionen und Grundsätze der Europäischen Datenschutzrichtlinie auf die Dienste und das Geschäftsmodell von Anbietern Sozialer Netzwerke im Internet detailliert geprüft wurde. Das Ergebnis ist positiv und wird in einer übersichtlichen Zusammenfassung der Pflichten des Anbieters und der Rechte des Nutzers angeboten.

Zur Anwendbarkeit von EG-Richtlinien wird festgestellt, dass die Bestimmungen der Datenschutzrichtlinie im Allgemeinen auch dann angewendet werden können, wenn sich der Hauptsitz des Anbieters außerhalb der EU befindet. Diensteanbieter und auch beteiligte Anwendungsanbieter sind im Sinne der Richtlinie verantwortlich für die Verarbeitung der Daten. Einer besonderen Diskussion ist wert, dass der Nutzer des Sozialen Netzwerks zuneh- mend mehr eigene Verantwortung für die Datenverarbeitung in seinem eigenen Umfeld übernimmt. Beispiele dafür sind die Nutzung von Daten aus Webservices, der Umgang mit Profilen von Networking-Partnern oder die gezielte Weiterverarbeitung von Daten Dritter. Diese Rolle des Nutzers ist durch die Richtlinie nicht abgedeckt. Für die Anbieter von Sozialen Netzwerken ergeben sich somit richtlinienkonforme Verpflichtungen:

- Der Anbieter muss dem Nutzer eine juristisch verbindliche Anbieterkennzeichnung bieten und für umfassende und klare Informationen über die Zwecke und Arten der beabsichtigten Verarbeitung von erhobenen persönlichen Daten sorgen;

- hat für seine Dienste Standardeinstellungen anzubieten, die die Privatsphäre bestmöglich schützen;

- soll die Nutzer vor der Abgabe persönlicher Daten an den Dienstleister über Risiken aufklären und angemessen warnen;

- soll die Nutzer darauf hinweisen, dass sie Daten anderer Personen nur mit deren Zustimmung abgegeben sollten;

- soll auf seiner Homepage für Nutzer und Dritte eine Beschwerdemöglichkeit für Datenschutzprobleme anbieten;

- soll einen definierten Zeitraum benennen, nach dem die Daten inaktiver Benutzer ebenso wie nicht mehr aktive Konten gelöscht werden.

$\mathrm{Zu}$ den Rechten von Benutzern wird festgestellt:

- Sowohl Mitglieder von Sozialen Netzwerken als auch Nicht-Mitglieder haben die Rechte von betroffenen Personen im Sinne der Art. 10-14 der EG-Datenschutz-Richtlinie;

- Sowohl Mitglieder als auch Nicht-Mitglieder sollten Zugang zu einem benutzerfreundlichen - vom Diensteanbieter eingerichteten - Beschwerdeverfahren haben;

- Benutzer sollten im Allgemeinen ein Pseudonym annehmen dürfen. 